УДК 681.518

\title{
The Method of Estimating the Optimal Composition of Forces and Means in Repelling Air Attack
}

\author{
Vladimir V. Tolstykh*a and Sergey V. Golubev ${ }^{\mathrm{b}}$ \\ ${ }^{a}$ Military Academy of the General Staff \\ of the Armed Forces of the Russian Federation \\ 100 Vernadsky, Moscow, 119571, Russia \\ ${ }^{b}$ Military Education and Research Centre of Military-Air Forces \\ «Military-Air Academy \\ Named After Professor N.E. Zhukovsky and Yu.A. Gagarin» \\ 54a Starykh Bolshevikov Str., Voronezh, 394064, Russia
}

Received 16.03.2017, received in revised form 19.03.2017, accepted 07.04.2017

The offered method of estimating the optimal composition of forces and means in the reflection of massive air raids on the basis of matrix performance using multiple evaluation criteria of the outcome of the result.

Keywords: military control systems, criterias of choice, efficiency matrix.

Citation: Tolstykh V.V., Golubev S.V. The model estimates the optimal composition of forces and means in repelling air attack, J. Sib. Fed. Univ. Eng. technol., 2017, 10(4), 450-456. DOI: 10.17516/1999-494X-2017-10-4-450-456.

(c) Siberian Federal University. All rights reserved

* Corresponding author E-mail address: oficer.1978@mail.ru 


\title{
Способ оценки оптимального состава сил и средств
}

\section{при отражении авиационного удара}

\author{
В.В. Толстых ${ }^{\mathbf{a}}$, С.В. Голубев ${ }^{\sigma}$ \\ ${ }^{a}$ Военная академия Генерального штаба \\ Вооруженных Сил Российской Федерации \\ Россия, 119571, Москва, пр. Вернадского, 100 \\ ${ }^{6}$ Военный учебно-научный центр Военно-воздушных сил \\ «Военно-воздушная академия \\ имени профессора Н.Е. Жуковского и Ю.А. Гагарина» \\ Россия, 394064, Воронеж, ул. Старых Большевиков, 54 а
}

Предлагается способ оценки оптимального состава сил и средств при отражении массированного авиационного удара на основе матрицы эффективности с использованием множества критериев оценки исхода результата.

Ключевые слова: военные системы управления, критерии выбора, матрица эффективности.

На текущий момент существующие военные системы управления как инструмент, позволяющий оптимально применять средства огневых воздействий при решении задач отражения массированного авиационного удара, всегда оперируют с неполной исходной информацией. Эти обстоятельства ведут к тому, что средства оценки эффективности результатов применения того или иного варианта не могут опираться на детерминированные способы построения этого прогноза. В связи с этим предлагается использовать матрицу эффективности на основе множества критериев оценки исхода результата.

Специфические черты систем управления часто не позволяют свести операции, проводимые этими системами, к детерминированным или вероятностным.

Условия оценки эффективности вариантов применения сил и средств в условиях неполной исходной информации отражены в табл. 1, в которой обозначены:

- $a_{i}$. вектор управляемых параметров, определяющий свойства системы;

- $n_{j}$ - вектор неуправляемых параметров, определяющий состояние обстановки (в нашем случае этим могут выступать действия противника);

- $k_{i j}$ - значение эффективности системы $a_{i}$ для состояния обстановки $n_{j}$;

- $K\left(a_{i}\right)$ - эффективность системы (варианта) $a_{i}$.

Каждая строка таблицы содержит значения эффективности одной системы для всех состояний обстановки n, а каждый столбец - значения эффективности для всех систем $a$ при одном и том же состоянии обстановки $[1,2]$.

В неопределенной операции могут быть известны множество состояний обстановки и эффективность систем для каждой из них, но нет данных, с какой вероятностью может появиться то или иное состояние.

Единого критерия оценки эффективности для неопределенных ситуаций не существует. Наиболее часто в неопределенных ситуациях используются критерии:

- среднего выигрыша; 
Таблица 1. Матрица эффективности

\begin{tabular}{|c|c|c|c|c|c|}
\hline \multirow{2}{*}{$a_{i}$} & \multicolumn{4}{|c|}{$\mathrm{n}_{\mathrm{j}}$} & \multirow{2}{*}{$\mathrm{K}\left(\mathrm{a}_{\mathrm{i}}\right)$} \\
\hline & $\mathrm{n}_{1}$ & $\mathrm{n}_{2}$ & $\ldots$ & $\mathrm{n}_{\mathrm{k}}$ & \\
\hline$a_{1}$ & $\mathrm{k}_{11}$ & $\mathrm{k}_{12}$ & $\ldots$ & $\mathrm{k}_{\mathrm{lk}}$ & \\
\hline$a_{2}$ & $\mathrm{k}_{21}$ & $\mathrm{k}_{22}$ & $\ldots$ & $\mathrm{k}_{2 \mathrm{k}}$ & \\
\hline$\ldots$ & $\ldots$ & $\ldots$ & $\ldots$ & $\ldots$ & \\
\hline$a_{m}$ & $\mathrm{k}_{\mathrm{m} 1}$ & $\mathrm{k}_{\mathrm{m} 2}$ & $\ldots$ & $\mathrm{k}_{\mathrm{mk}}$ & \\
\hline
\end{tabular}

Таблица 2. Исходная матрица с результатами частных исходов

\begin{tabular}{|c|c|c|c|c|}
\hline \multirow{2}{*}{$\mathrm{a}_{\mathrm{i}}$} & \multicolumn{4}{|c|}{$\mathrm{n}_{\mathrm{j}}$} \\
\cline { 2 - 5 } & $\mathrm{n}_{1}$ & $\mathrm{n}_{2}$ & $\mathrm{n}_{3}$ & $\mathrm{n}_{4}$ \\
\hline $\mathrm{a}_{1}$ & 0.1 & 0.5 & 0.1 & 0.2 \\
\hline $\mathrm{a}_{2}$ & 0.2 & 0.3 & 0.2 & 0.4 \\
\hline $\mathrm{a}_{3}$ & 0.1 & 0.4 & 0.4 & 0.3 \\
\hline
\end{tabular}

- Лапласа;

- осторожного наблюдателя (Вальда);

- максимакса;

- пессимизма-оптимизма (Гурвица);

- минимального риска (Сэвиджа) [2].

Рассмотрим возможность применения матрицы эффективности для оценки исходов вариантов действия при применении авиационных комплексов при отражении воздушных целей. Для этого $\mathrm{n}_{1}$ - одна воздушная цель, $\mathrm{n}_{2}$ - две воздушные цели, $\mathrm{n}_{3}-$ три воздушные цели, $\mathrm{n}_{4}-$ четыре воздушные цели. Матрица эффективности представлена в табл. 2.

\section{Критерий среднего вылгрыша}

Данный критерий предполагает задание вероятностей состояния обстановки $\mathrm{p}_{\mathrm{i}}$. Эффективность систем оценивается как среднее значение оценок эффективности по всем состояниям обстановки $[2,3]$ :

$$
K\left(a_{i}\right)=\sum_{j=1}^{t} p_{j} k_{i j},
$$

$\mathrm{K}_{\text {опт }}=\max \mathrm{K}\left(\mathrm{a}_{\mathrm{i}}\right)$.

Если $\mathrm{p}_{1}=0.4, \mathrm{p}_{2}=0.2, \mathrm{p}_{3}=0.1, \mathrm{p}_{4}=0.3$, то получим:

$$
K\left(a_{1}\right)=0.21, K\left(a_{2}\right)=0.28, K\left(a_{3}\right)=0.25 \text {. }
$$

Следовательно, вероятность того, что появится в зоне ответственности одна цель, $\mathrm{p}_{\mathrm{l}}=0.4$, две цели $-\mathrm{p}_{2}=0.2$, три цели $-\mathrm{p}_{3}=0.1$, четыре цели $-\mathrm{p}_{4}=0.3$. После обработки результатов эффективность использования первого варианта $=0.21$, второго варианта $=0.28$, третьего вариан$\mathrm{Ta}=0.25$. 
Оптимальное решение - вариант $a_{2}$.

Критерий Лапласа

В основе критерия лежит предположение: поскольку о состояниях обстановки ничего не известно, то их можно считать равновероятными.

$$
K\left(a_{i}\right)=\frac{1}{n} \sum_{j=1}^{t} k_{i j},
$$

$\mathrm{K}_{\text {опт }}=\max \mathrm{K}\left(\mathrm{a}_{\mathrm{i}}\right)$.

Рассчитаем эффективность систем по данному критерию для приведенного примера:

$$
\begin{aligned}
& K\left(a_{1}\right)=0,25(0,1+0,5+0,1+0,2)=0,225 ; \\
& K\left(a_{2}\right)=0,25(0,2+0,3+0,2+0,4)=0,275 ; \\
& K\left(a_{3}\right)=0,25(0,1+0,4+0,4+0,3)=0,3 .
\end{aligned}
$$

Оптимальное решение - вариант $a_{3}$. Критерий Лапласа представляет собой частный случай критерия среднего выигрыша.

\section{Критерий осторожного наблюдателя (Вальда)}

Это максиминный критерий, он гарантирует определенный выигрыш при наихудших условиях. Критерий основывается на том, что, если состояние обстановки неизвестно, нужно поступать самым осторожным образом, ориентируясь на минимальное значение эффективности каждой системы.

В каждой строке матрицы эффективности находится минимальная из оценок систем по различным состояниям обстановки. Оптимальной считается система из строки с максимальным значением эффективности.

$$
K\left(a_{i}\right)=\min k_{i j},
$$

$\mathrm{K}_{\text {опт }}=\max \mathrm{K}\left(\mathrm{a}_{\mathrm{i}}\right)$.

Применение критерия максимина к нашему примеру дает следующие оценки [2]:

$$
\begin{aligned}
& K\left(a_{1}\right)=\min (0,1 ; 0,5 ; 0,1 ; 0,2)=0,1 ; \\
& K\left(a_{2}\right)=\min (0,2 ; 0,3 ; 0,2 ; 0,4)=0,2 ; \\
& K\left(a_{3}\right)=\min (0,1 ; 0,4 ; 0,4 ; 0,3)=0,1 .
\end{aligned}
$$

Оптимальное решение - вариант $a_{2}$.

Максиминный критерий ориентирует на решение, не содержащее элементов риска: при любом из возможных состояний обстановки выбранная система покажет результат операции не хуже найденного максимина. Такая осторожность является в ряде случаев недостатком критерия. Другой недостаток - добавление постоянного числа к каждому элементу столбца матрицы эффективности влияет на выбор системы. 


\section{Критерий максимакса}

Этим критерием предписывается оценивать системы по максимальному значению эффективности и выбирать в качестве оптимального решения систему, обладающую эффективностью с наибольшим из максимумов $[2,3]$.

$$
K\left(a_{i}\right)=\max k_{i j}
$$

$\mathrm{K}_{\text {опт }}=\max \mathrm{K}\left(\mathrm{a}_{\mathrm{i}}\right)$.

Оценки систем на основе максимаксного критерия в нашем примере принимают такие значения:

$$
\begin{aligned}
& K\left(a_{1}\right)=\max (0,1 ; 0,5 ; 0,1 ; 0,2)=0,5 ; \\
& K\left(a_{2}\right)=\max (0,2 ; 0,3 ; 0,2 ; 0,4)=0,4 ; \\
& K\left(a_{3}\right)=\max (0,1 ; 0,4 ; 0,4 ; 0,3)=0,4 .
\end{aligned}
$$

Оптимальное решение - вариант $a_{1}$. Критерий максимакса - самый оптимистический критерий. Те, кто предпочитает им пользоваться, всегда надеются на лучшее состояние обстановки и, естественно, в большой степени рискуют.

\section{Критерий пессимизма-оптимизма (Гурвица)}

Это критерий обобщенного макси/мини. Согласно данному критерию при оценке и выборе систем неразумно проявлять как осторожность, так и азарт, а следует, учитывая самое высокое и самое низкое значения эффективности, занимать промежуточную позицию (взвешиваются наихудшие и наилучшие условия). Для этого вводится коэффициент оптимизма $\alpha(\mathrm{O}<\alpha<1)$, характеризующий отношение к риску лица, принимающего решение. Эффективность систем находится как взвешенная с помощью коэффициента $\alpha$ сумма максимальной и минимальной оценок [2]:

$$
K\left(a_{i}\right)=\alpha \max k_{i j}+(1-\alpha) \min k_{i j} .
$$

Условие оптимальности записывается в виде

$$
K_{\text {onm }}=\max K\left(a_{i}\right), 0<\alpha<1 .
$$

Зададимся значением $\alpha=0,6$ и рассчитаем эффективность систем для рассматриваемого примера:

$$
\begin{aligned}
& K\left(a_{1}\right)=0,34 ; \\
& K\left(a_{2}\right)=0,32 ; \\
& K\left(a_{3}\right)=0,34 .
\end{aligned}
$$

Оптимальным вариантом будет $a_{1}$.

Значение $\alpha$ может определяться методом экспертных оценок. Очевидно, чем опаснее оцениваемая ситуация, тем ближе величина $\alpha$ должна быть к единице, когда гарантируется наибольший из минимальных выигрышей или наименьший из максимальных рисков. 
На практике пользуются значениями коэффициента $\alpha$ в пределах $0,3-0,7$.

\section{Критерий минимального риска (Сэвиджа)}

Минимизирует потери эффективности при наихудших условиях. Для оценки систем на основе данного критерия матрица эффективности должна быть преобразована в матрицу потерь (риска). Каждый элемент матрицы потерь определяется как разность между максимальным и текущим значениями оценок эффективности в столбце [1]:

$$
\Delta k_{i j}=\max _{i} k_{i j}-k_{i j}
$$

После преобразования матрицы используется критерий минимакса:

Оценим эффективность систем из приведенного примера в соответствии с данным критерием. Матрице эффективности будет соответствовать матрица потерь (табл. 3). Тогда

$$
K\left(a_{1}\right)=0,3 ; K\left(a_{2}\right)=0,2 ; K\left(a_{3}\right)=0,1 .
$$

Оптимальное решение - вариант $\mathrm{a}_{1}$. Критерий минимального риска отражает сожаление по поводу того, что выбранная система не оказалась наилучшей при определенном состоянии обстановки. Так, если произвести выбор системы $a_{l}$, а состояние обстановки в действительности $\mathrm{n}_{3}$, то сожаление, что не выбрана наилучшая из систем $\left(a_{3}\right)$, составит 0,3 . О критерии Сэвиджа можно сказать, что он, как и критерий Вальда, относится к числу осторожных критериев. По сравнению с критерием Вальда в нем придается несколько большее значение выигрышу, чем проигрышу.

Таким образом, эффективность систем в неопределенных операциях может оцениваться по целому ряду критериев. На выбор того или иного критерия оказывает влияние ряд факторов:

- природа конкретной операции и ее цель (в одних операциях допустим риск, в других нужен гарантированный результат);

- причины неопределенности (одно дело, когда неопределенность является случайным результатом действия объективных законов природы, и другое, когда она вызывается действиями разумного противника, стремящегося помешать в достижении цели);

- характер лица, принимающего решение (одни люди склонны к риску в надежде добиться большего успеха, другие предпочитают действовать всегда осторожно).

Устойчивость выбранного рационального варианта можно оценить на основе анализа по нескольким критериям. Если существует совпадение, то имеется большая уверенность в правильности выбора варианта.

Таблица 3. Матрица потерь

\begin{tabular}{|c|c|c|c|c|}
\hline \multirow{2}{*}{$\mathrm{a}_{\mathrm{i}}$} & \multicolumn{4}{|c|}{$\mathrm{n}_{\mathrm{j}}$} \\
\cline { 2 - 5 } & $\mathrm{n}_{1}$ & $\mathrm{n}_{2}$ & $\mathrm{n}_{3}$ & $\mathrm{n}_{4}$ \\
\hline $\mathrm{a}_{1}$ & 0.1 & 0 & 0.3 & 0.2 \\
\hline $\mathrm{a}_{2}$ & 0 & 0.2 & 0.2 & 0 \\
\hline $\mathrm{a}_{3}$ & 0.1 & 0.1 & 0 & 0.1 \\
\hline
\end{tabular}




\section{Список литературы}

[1] Айзерман М.А., Алескеров Ф.Т. Выбор вариантов (основы теории). М.: Наука, 1990. 236 c. [Ayzerman M.A., Aleskerov F.T. Choice of variants (theory basis). Moscow, Nauka, 1990, 236 p. (in Russian)]

[2] Ларичев О.И. Теория и методы принятия решений. М.: Логос, 2000. 296 c. [Larichev O.I. The theory and decision-making methods. Moscow, Logos, 2000, 296 p. (in Russian)]

[3] Науман Э. Принять решение - но как? М.: Мир, 1987. 198 с. [Nauman Е. To make the decision - but how? Moscow, Mir, 1987, 198 p. (in Russian)] 